\title{
Evolutionary and asymptotic stability in symmetric multi-player games
}

\author{
Maciej Bukowski \\ Institute of Economics \\ Warsaw School of Economics \\ Aleje Niepodległości 162 \\ 02-554 Warsaw, Poland \\ e-mail: mbukows@sgh.waw.pl \\ and \\ Jacek Miȩkisz \\ Institute of Applied Mathematics and Mechanics \\ Warsaw University \\ ul. Banacha 2 \\ 02-097 Warsaw, Poland \\ e-mail: miekisz@mimuw.edu.pl
}

October 27, 2018

\begin{abstract}
We provide a classification of symmetric three-player games with two strategies and investigate evolutionary and asymptotic stability (in the replicator dynamics) of their Nash equilibria. We discuss similarities and differences between two-player and multi-player games. In particular, we construct examples which exhibit a novel behavior not found in two-player games.
\end{abstract}

Key Words: Multi-player games, evolutionarily stable strategies, asymptotic stability, replicator dynamics, risk-dominance. 


\section{Introduction}

Equilibrium behavior of systems of many interacting entities can be described in the framework of game-theoretic models. Although there are many players in these models, their strategic interactions are usually decomposed into a sum of two-player games $[1,2,3,4,5]$. Only recently, there have appeared some systematic studies of truly multi-player games $[6,7,8]$.

We will provide here a classification of symmetric three-player games with two strategies and describe their symmetric Nash equilibria and evolutionarily stable strategies. As in two-player games, for a certain range of payoff parameters, there exist multiple Nash equilibria.

In the first class of our games, there are two pure Nash equilibria and a mixed one. Such games are three-player analogs of two-player coordination games. In the second class, there are two mixed Nash equilibria and a pure one. One may also have games with one pure and one mixed Nash equilibrium. We are faced therefore with a standard problem of equilibrium selection. We discuss in this context evolutionarily stable strategies. We develop a simple criterion to check whether a given Nash equilibrium is evolutionarily stable in a game with two strategies and apply it to three-player games. Then we investigate the asymptotic stability of Nash equilibria in the replicator dynamics. Here we also encounter some novel behavior. It concerns supersymmetric games, i.e., those where for any given profile of strategies, payoffs of all players are the same. Any symmetric $n$-player game with two strategies can be transformed by the standard payoff transformation into a supersymmetric game which has the same set of Nash equilibria and evolutionarily stable strategies $[9,10,11]$. In other words, any $n$-player game with two strategies is a so called potential game [12]. It is known that in two-player games, interior evolutionarily stable strategies are globally asymptotically stable. Three-player games with two strategies belonging to the second category of our classification have two evolutionarily stable strategies, a pure and an interior one. Due to a general theorem, they are both asymptotically stable and therefore the interior one is not globally stable. We also construct an example of 
a four-player supersymmetric game with two interior evolutionarily stable strategies, hence

neither of them is globally stable. It is also known that in supersymmetric two-player games, a strategy is evolutionarily stable if and only if it is asymptotically stable in the replicator dynamics. We show that this is also true in supersymmetric n-player games.

Finally, we discuss the concept of risk-dominance [13] and its relation to the size of the basin of attraction in the replicator dynamics of a Nash equilibrium. We show, that unlike in two-player games with two strategies, risk-dominant strategies may have smaller basins of attraction than dominated ones.

In Section 2, we define multi-player games. In Section 3, we provide a classification of symmetric three-player games with two strategies and describe their symmetric Nash equilibria. In Section 4, we discuss evolutionarily stable strategies of our games. In Section 5, we study asymptotic stability of Nash equilibria in the replicator dynamics. In Section 6, relations between the risk-dominance and the size of the basin of attraction are analyzed. Discussion follows in Section 7.

\section{Multi-Player Games}

Definition $1 A$ game in the normal form is a triple $G=(I, S, \pi)$, where $I=\{1,2, \ldots, n\}$ is the set of players, $S=\times_{i=1}^{n} S_{i}$, where $S_{i}=\left\{1,2, \ldots, k_{i}\right\}$, is the finite set of strategies available for each player, $\pi: S \rightarrow R^{n}$ is a payoff function assigning to every profile of pure strategies, $s=\left(s_{1}, s_{2}, \ldots, s_{n}\right) \in S$, a vector of payoffs, $\pi(s)=\left(\pi_{1}(s), \pi_{2}(s), \ldots \pi_{n}(s)\right)$, where $\pi_{i}(s)$ is the payoff of the $i$-th player in the profile $s$.

We will consider here only symmetric games. In such games, all players assume the same role in the game and moreover the payoff of any player depends only on his strategy and numbers of players playing different types of strategies. More formally,

Definition $2 G$ is called symmetric, if for every permutation of the set of players, $\sigma: I \rightarrow I$, 
we have $\pi_{i}\left(s_{1}, s_{2}, \ldots, s_{n}\right)=\pi_{\sigma^{-1}(i)}\left(s_{\sigma(1)}, s_{\sigma(2)}, \ldots, s_{\sigma(n)}\right)$.

A special subclass of symmetric games consist of supersymmetric games, where all players get the same payoff dependent only on the profile of strategies.

Definition $3 G=(I, S, \pi)$ is called supersymmetric if for every permutation of the set of players, $\sigma: I \rightarrow I$, and every $i, \pi_{i}\left(s_{1}, s_{2}, \ldots, s_{n}\right)=\pi_{1}\left(s_{\sigma(1)}, s_{\sigma(2)}, \ldots, s_{\sigma(n)}\right)$.

Payoffs in symmetric games are uniquely determined by the payoff function of, say, the first player: $u_{1}: S \rightarrow R$. We set $a_{s_{1} s_{2} \ldots s_{n}}=\pi_{1}\left(s_{1}, s_{2}, \ldots, s_{n}\right)$.

The payoff function of a two-player game with $k$ strategies can be then represented by a $k \times k$ matrix, $A=\left(a_{i j}\right)$, where $a_{i j}$ is the payoff of the first (row) player when he plays the strategy $i$, while the second (column) player is playing the strategy $j$.

Payoffs of a three-player game with $k$ strategies can be represented by $k$ such matrices.

$$
A=\left(A_{1}, A_{2}, \ldots, A_{k}\right)
$$

where

$$
\forall_{j \in\{1,2, \ldots, k\}} \quad A_{j}=\left(\begin{array}{cccc}
a_{11 j} & a_{12 j} & \ldots & a_{1 k j} \\
a_{21 j} & a_{22 j} & \ldots & a_{2 k j} \\
\ldots & & & \\
a_{k 1 j} & a_{k 2 j} & \ldots & a_{k k j}
\end{array}\right)
$$

is a payoff matrix of the row player when the third (matrix) player plays the strategy $j$.

In particular, in the case of a three-player game with two strategies

$$
A=\left(\left(\begin{array}{ll}
a_{111} & a_{121} \\
a_{211} & a_{221}
\end{array}\right),\left(\begin{array}{ll}
a_{112} & a_{122} \\
a_{212} & a_{222}
\end{array}\right)\right)
$$

Payoffs in a four-player game with two strategies are given by

$$
A=\left(\left(\left(\begin{array}{ll}
a_{1111} & a_{1211} \\
a_{2111} & a_{2211}
\end{array}\right),\left(\begin{array}{ll}
a_{1121} & a_{1221} \\
a_{2121} & a_{2221}
\end{array}\right)\right),\left(\left(\begin{array}{ll}
a_{1112} & a_{1212} \\
a_{2112} & a_{2212}
\end{array}\right),\left(\begin{array}{ll}
a_{1122} & a_{1222} \\
a_{2122} & a_{2222}
\end{array}\right)\right)\right)
$$


A mixed strategy of the $i$-th player will be denoted by $x_{i}$ and the set of all mixed strategies by $\Delta_{i}$

$$
\Delta_{i}=\left\{x_{i} \in R^{k}: x_{i}=\left\{x_{i 1}, x_{i 2}, \ldots, x_{i k}\right\}, \text { where } x_{i m} \geq 0 \text { and } \sum_{m=1}^{k} x_{i m}=1\right\} .
$$

A pure strategy $m$ is sometimes denoted by $e^{m}=(0, \ldots, 1, \ldots, 0)$. The support of a mixed strategy $x_{i}$ is the set of pure strategies played with nonzero probabilities,

$$
\operatorname{supp}\left(x_{i}\right)=\left\{m \in S_{i}: x_{i m}>0\right\}
$$

$\Theta=\times_{i=1}^{n} \Delta_{i}$ is the set of profiles of mixed strategies.

If $s=\left\{s_{1}, \ldots, s_{n}\right\}$ and $x \in \Theta$, then $x(s)=\prod_{i=1}^{n} x_{i s_{i}}$ and we set the expected payoff for the $i$-th player

$$
u_{i}(x)=\sum_{s \in S} x(s) \pi_{i}(s)
$$

Definition $4 x \in \Theta$ is a Nash equilibrium if $\forall_{i \in I, z_{i} \in \Delta_{i}}$

$$
u_{i}\left(x_{i}, x_{-i}\right) \geq u_{i}\left(z_{i}, x_{-i}\right)
$$

where $x_{-i} \in \times_{j \neq i} \Delta_{j}$ is a profile of strategies of all players except the $i$-th one.

The set of Nash equilibria of any game with finite number of players and strategies is nonempty and is denoted by $\Theta^{N E}$.

Here we consider only symmetric Nash equilibria, that is elements of

$$
\Delta^{N E}=\left\{x \in \Delta:\left(x, x^{n-1}\right) \in \Theta^{N E}\right\}
$$

where $\Delta$ is the simplex of mixed strategies common for all players and $\left(x, x^{n-1}\right)=(x, x, \ldots, x)$. The payoff $u_{i}\left(x, x^{n-1}\right)$ is the same for all $i$ and we denote it by $u\left(x, x^{n-1}\right)$.

We have the following useful characterization of symmetric mixed Nash equlibria.

Theorem 1 In a symmetric game, if $x \in \Delta^{N E}$, then $\forall_{m \in \operatorname{supp}(x)} u\left(e^{m}, x^{n-1}\right)=u\left(x, x^{n-1}\right)=$ const. If $x \in$ int $\Delta$, then $x \in \Delta^{N E}$ if and only if $\forall_{m} u\left(e^{m}, x^{n-1}\right)=u\left(x, x^{n-1}\right)=$ const. 
Proof: If $x \in \Delta^{N E}$, then $u\left(e^{m}, x^{n-1}\right) \leq u\left(x, x^{n-1}\right)$ for all $m$. If there existed $m \in \operatorname{supp}(x)$ such that $u\left(e^{m}, x^{n-1}\right)<u\left(x, x^{n-1}\right)$, then

$$
u\left(x, x^{n-1}\right)=\sum_{m \in \operatorname{supp}(x)} x_{i} u\left(e^{m}, x^{n-1}\right)<\sum_{m \in \operatorname{supp}(x)} x_{i} u\left(x, x^{n-1}\right)=u\left(x, x^{n-1}\right)
$$

which is not possible. Similarly, if $x \in$ int $\Delta$ and $\forall_{m} u\left(e^{m}, x^{n-1}\right)=u\left(x, x^{n-1}\right)$, then $\forall_{y \in \Delta}$

$$
u\left(y, x^{n-1}\right)=\sum_{m \in \operatorname{supp}(y)} y_{i} u\left(e^{m}, x^{n-1}\right)=\sum_{m \in \operatorname{supp}(y)} y_{i} u\left(x, x^{n-1}\right)=u\left(x, x^{n-1}\right)
$$

which finishes the proof.

\section{Nash Equilibria in Three-Player Games with Two Strate- gies}

In this section, we completely characterize symmetric Nash equilibria in symmetric three-player games with two strategies. Without loss of generality (adding payoffs to columns of payoff matrices does not change the incentive functions and therefore neither Nash equilibria nor their stability properties in the replicator dynamics) we will consider games defined by the following generic payoff function:

$$
A=\left(\left(\begin{array}{ll}
a & 0 \\
0 & b
\end{array}\right),\left(\begin{array}{ll}
0 & 0 \\
b & c
\end{array}\right)\right)
$$

We may also add $b$ to the fourth column to obtain a supersymmetric game with the payoff function

$$
A^{\prime}=\left(\left(\begin{array}{ll}
a & 0 \\
0 & b
\end{array}\right),\left(\begin{array}{cc}
0 & b \\
b & c+b
\end{array}\right)\right)
$$

Both $A$ and $A^{\prime}$ have the same family of Nash equilibria.

It follows that $(1,0) \in \Delta^{N E}$ if and only if $a \geq 0$ and $(0,1) \in \Delta^{N E}$ if and only if $c \geq 0$. Now we will investigate symmetric interior (mixed) Nash equilibria. In games with two strategies we will identify $x \in \Delta$ with the probability of playing the first strategy. Theorem 1 tells us 
that an interior point $(x, 1-x)$ of the simplex $\Delta$ is a symmetric Nash equilibrium if and only if

$$
a x^{2}=2 b x(1-x)+c(1-x)^{2}
$$

which is equivalent to the following equation:

$$
(a+2 b-c) x^{2}-2(b-c) x-c=0
$$

If $b=\frac{(c-a)}{2}$, then the above equation is reduced to

$$
(a+c) x=c
$$

Hence if $a=-c \neq 0$, then there are no interior symmetric Nash equlibria, if $a=b=c=0$, then every point is a Nash equilibrium and finally if $a \neq-c$ and $a c>0$, then $x=\frac{c}{a+c}$ is an interior Nash equilibrium. Now we will consider the case when $w(x)$ is a quadratic function. Let us notice that

$$
\begin{array}{ll}
\text { i) } & w(0)=-c \text { and } w(1)=a \\
\text { ii) } & w^{\prime}\left(x_{e}\right)=0 \Leftrightarrow x_{e}=\frac{b-c}{a+2 b-c} \\
\text { iii) } & w\left(x_{e}\right)=-\frac{b^{2}+a c}{a+2 b-c}
\end{array}
$$

where $w^{\prime}(x)$ is the derivative of $w(x)$. From $(i)$ we have that if $a c>0$, then $w(x)$ has a unique root in the interval $(0,1)$ hence there exists a unique symmetric interior Nash equilibrium. However, if $a c<0$, then an interior symmetric Nash equilibrium exists if and only if the following conditions are satisfied:

$$
\begin{aligned}
& \text { i) } 0<x_{e}<1 \\
& \text { ii) } \quad w(0) w\left(x_{e}\right)=c \frac{b^{2}+a c}{a+2 b-c} \leq 0 .
\end{aligned}
$$

From $(i)$ we obtain that either $b>\max (c,-a)=-\min (-c, a)$ or $b<\min (c,-a)=-\max (-c, a)$, which together with $(i i)$ gives us that if $a c<0$, then a symmetric interior Nash equilibrium exists if and only if one of two following conditions is satisfied:

$$
\begin{aligned}
& \text { a) } \quad c<0 \text { and } b \geq \sqrt{|a c|} \\
& \text { b) } \quad c>0 \text { and } b \leq-\sqrt{|a c|}
\end{aligned}
$$


Moreover, if $|b|=\sqrt{|a c|}$, then there exists a unique symmetric interior Nash equilibrium and if $|b|>\sqrt{|a c|}$, then there are two such Nash equilibria. Now we have to deal with the case $a c=0$. If $a=0$, then $w(x)$ has the following form:

$$
\begin{gathered}
w(x)=x^{2}(2 b-c)-2 x(b-c)-c \\
w^{\prime}(x)=2 x(2 b-c)-2(b-c)
\end{gathered}
$$

which means that $w(1)=0$ and $w^{\prime}(1)=2 b$ hence $w(x)$ has a root in $(0,1)$ if and only if $b c<0$. If $c=0$, then $w(x)$ is reduced to:

$$
\begin{aligned}
w(x) & =x^{2}(a+2 b)-2 b x, \\
w^{\prime}(x) & =2 x(a+2 b)-2 b
\end{aligned}
$$

and it follows that $w(0)=0$ and $w^{\prime}(0)=-2 b$ hence $w(x)$ has a root in $(0,1)$ if and only if $a b>0$. We can sum up the above analysis in the following classification of three-player games with two strategies.

Category I: Games with three symmetric (two pure and one mixed) Nash equilibria. $\Delta^{N E}=\{(1,0),(0,1),(x, 1-x)\}$ if $(a>0$ and $c>0)$ or $(a=0, b<0$, and $c>0)$ or $(a>0, b>0$, and $c=0)$. In the first case, if $b \neq \frac{(c-a)}{2}$, then $x=\frac{(b-c)+\sqrt{b^{2}+a c}}{(a+2 b-c)}$ and if $b=\frac{(c-a)}{2}$, then $x=\frac{c}{a+c}$. In the second case, $x=\frac{-c}{2 b-c}$. In the third case, $x=\frac{2 b}{2 b+a}$.

Category II: Games with three symmetric (one pure and two mixed) Nash equilibria.

1. $\Delta^{N E}=\left\{(1,0),\left(x_{1}, 1-x_{1}\right),\left(x_{2}, 1-x_{2}\right)\right\}$, where $x_{1}=\frac{(b-c)-\sqrt{b^{2}+a c}}{(a+2 b-c)}$ and $x_{2}=\frac{(b-c)+\sqrt{b^{2}+a c}}{(a+2 b-c)}$, if $a>0, c<0$ and $b>\sqrt{|a c|}$.

2. $\Delta^{N E}=\left\{(0,1),\left(x_{1}, 1-x_{1}\right),\left(x_{2}, 1-x_{2}\right)\right\}$, where $x_{1}$ and $x_{2}$ are as before, if $a<0, c>0$ and $b<-\sqrt{|a c|}$.

Category III: Games with one pure and one mixed Nash equilibrium. 
1. $\Delta^{N E}=\{(1,0),(x, 1-x)\}$ if $(a>0, c<0$, and $b=\sqrt{|a c|})$ or $(a=0, b>0$, and $c<0)$. In the first case, $x=\frac{\sqrt{|c|}}{\sqrt{a}+\sqrt{|c|}}$. In the second case, $x=\frac{-c}{2 b-c}$.

2. $\Delta^{N E}=\{(0,1),(x, 1-x)\}$ if $(a<0, c>0$ and $b=-\sqrt{|a c|})$ or $(a<0, b<0$, and $c=0)$. In the first case, $x=\frac{\sqrt{c}}{\sqrt{|a|}+\sqrt{c}}$. In the second case, $x=\frac{2 b}{2 b+a}$.

Category IV: Games with one mixed Nash equilibrium.

$\Delta^{N E}=\{(x, 1-x)\}$ if $a<0$ and $c<0$. If $b=\frac{(c-a)}{2}$, then $x=\frac{c}{a+c}$ and if $b \neq \frac{(c-a)}{2}$, then $x=\frac{(b-c)-\sqrt{b^{2}+a c}}{(a+2 b-c)}$.

Category V: Games with two pure Nash equilibria.

$\Delta^{N E}=\{(1,0),(0,1)\}$ if $(a=0, b \geq 0$, and $c>0)$ or $(a>0, b \geq 0$, and $c=0)$ or $(a=0, b \neq 0)$, and $c=0$.

Category VI: Games with one pure Nash equilibrium.

1. $\Delta^{N E}=\{(1,0)\}$ if $(a>0, c<0$, and $b<\sqrt{|a c|})$ or $(a=0, b \leq 0$, and $c<0)$.

2. $\Delta^{N E}=\{(0,1)\}$ if $(a<0, c>0$, and $b>-\sqrt{|a c|})$ or $(a<0, b \leq 0$, and $c=0)$.

Category VII: Games with $\Delta^{N E}=\Delta$ for $a=0, b=0$, and $c=0$.

Let us notice that Categories III, IV, and VII contain nongeneric games.

In five of the above categories there are multiple Nash equilibria. We are therefore faced with the problem of equilibrium selection.

\section{Evolutionarily Stable Strategies}

We introduce the following definition of an evolutionarily stable strategy for n-player games $[7,8]$. 
Definition $5 x \in \Delta$ is an evolutionarily stable strategy (ESS) if there exists $0<\epsilon^{\prime}<1$ such that for all $0<\epsilon<\epsilon^{\prime}$ and for every $y \in \Delta$

$$
u\left(x,(\epsilon y+(1-\epsilon) x)^{n-1}\right)>u\left(y,(\epsilon y+(1-\epsilon) x)^{n-1}\right) .
$$

Definition $6 x \in \Delta$ is locally superior if there exists a neighborhood $U$ of $x$ such that for all $y \in U \cap \Delta$ we have

$$
u\left(x, y^{n-1}\right) \geq u\left(y, y^{n-1}\right)
$$

and the equality holds only if $y=x$.

The following theorem is a straightforward generalization of the corresponding theorem for two-player games [14].

Theorem $2 x \in \Delta^{E S S}$ if and only if $x$ is locally superior.

The above theorem can be used to obtain a useful criterion for evolutionary stability in the case of symmetric $n$-player games with two strategies [7]. Let $x, y \in \Delta$. Define an incentive function:

$$
w(y)=u\left(e^{1}, y^{n-1}\right)-u\left(e^{2}, y^{n-1}\right)
$$

Theorem 3 In symmetric n-player games with two strategies, $x \in \Delta^{E S S}$ if and only if for $y \in \Delta$ close to $x$ both of the following implications hold:

$$
\begin{aligned}
& \text { If } y<x, \text { then } w(y)>0 \\
& \text { If } y>x, \text { then } w(y)<0 .
\end{aligned}
$$

Proof: Consider the function

$$
w_{x}(y)=u\left(x-y, y^{n-1}\right)
$$


Due to Theorem 2, $x \in \Delta^{E S S}$ if and only if for any $y \in \Delta$ close to $x$ and $y \neq x$ we have $w_{x}(y)>0$. It follows that

$$
w_{x}(y)=(x-y)\left(u\left(e^{1}, y^{n-1}\right)-u\left(e^{2}, y^{n-1}\right)\right)
$$

which finishes the proof.

It follows that $x \in \Delta$ is evolutionarily stable if and only if $w(y)$ is a decreasing function in the neighborhood of $x$.

For $n \leq 3$ we have the following corollary.

Corollary $1 x \in \Delta^{E S S} \cap$ int $\Delta$ if and only if $w(x)=0$ and $w^{\prime}(x)<0$.

The following theorems concern the number and supports of evolutionarily stable strategies.

Theorem 4 In symmetric two-player games, if $x, y \in \Delta^{E S S}$ and $x \neq y$, then supp $(x) \nsubseteq$ $\operatorname{supp}(y)[15]$.

In particular, if $x \in \Delta^{E S S} \cap$ int $\Delta$, then $x$ is the unique evolutionarily stable strategy. The analogous theorem is not true in three-player games; games in Category I of our classification have one pure and one interior evolutionarily stable strategy. For three-player games we have the following weaker theorem.

Theorem 5 In symmetric three-player games, if $x, y \in \Delta^{E S S}$ and $x \neq y$, then $\operatorname{supp}(x) \neq$ $\operatorname{supp}(y)[7]$

It was already indicated in [7] that the above theorem cannot be generalized to games with $n \geq 4$ players. We illustrate it with the following example.

Example 1 A 4-player supersymmetric game with two interior evolutionarily stable strategies 
Payoffs are given by

$$
A=\left(\left(\left(\begin{array}{cc}
a_{1} & 0 \\
0 & a_{2}
\end{array}\right),\left(\begin{array}{cc}
0 & a_{3} \\
a_{2} & 0
\end{array}\right)\right),\left(\left(\begin{array}{cc}
0 & a_{3} \\
a_{2} & 0
\end{array}\right),\left(\begin{array}{cc}
a_{3} & 0 \\
0 & a_{4}
\end{array}\right)\right)\right)
$$

If $x \in \Delta^{N E} \cap$ int $\Delta$, then $x$ is a root of the equation $w(x)=0$, where

$$
w(x)=a_{1} x^{3}+3 a_{3} x(1-x)^{2}-3 a_{2} x^{2}(1-x)-a_{4}(1-x)^{3} .
$$

Put $a_{1}=a_{4}=-\frac{3}{32}$ and $a_{2}=a_{3}=-\frac{13}{96}$. Then:

$$
w(x)=-\left(x-\frac{1}{4}\right)\left(x-\frac{1}{2}\right)\left(x-\frac{3}{4}\right)
$$

It follows from Theorem 4 that both $\left(x_{1}, 1-x_{1}\right)=\left(\frac{1}{4}, \frac{3}{4}\right)$ and $\left(x_{2}, 1-x_{2}\right)=\left(\frac{3}{4}, \frac{1}{4}\right)$ are interior evolutionarily stable strategies.

\subsection{Evolutionarily stable strategies in three-player games with two strategies}

In this section, we completely characterize evolutionarily stable strategies in symmetric threeplayer games with two strategies. Again, without loss of generality we will consider games defined by the following generic payoff function:

$$
A=\left(\left(\begin{array}{ll}
a & 0 \\
0 & b
\end{array}\right),\left(\begin{array}{ll}
0 & 0 \\
b & c
\end{array}\right)\right)
$$

Due to Theorem 3, $x \in \Delta$ is ESS if and only if for all $y=(y, 1-y) \in \Delta$ close to $x$ the following conditions hold:

$$
\begin{aligned}
& y<x \Leftrightarrow a y^{2}-2 b y(1-y)-c(1-y)^{2}>0, \\
& y>x \Leftrightarrow a y^{2}-2 b y(1-y)-c(1-y)^{2}<0 .
\end{aligned}
$$

We will deal with the following polynomial:

$$
w(y)=y^{2}(a+2 b-c)-2 y(b-c)-c .
$$


$x \in \Delta$ is ESS if and only if for all $y$ close to $x$ and $y<x$ we have $w(y)>0$ and for $y>x$, $w(y)<0$. Checking the derivative of $w(y)$ we arrive at the following classification.

Category I: $\Delta^{E S S}=\{(1,0),(0,1)\}$ and $\Delta^{N E} \backslash \Delta^{E S S}=\{(x, 1-x)\}$.

\section{Category II:}

1. $\Delta^{E S S}=\left\{(1,0),\left(x_{1}, 1-x_{1}\right)\right\}$ and $\Delta^{N E} \backslash \Delta^{E S S}=\left\{\left(x_{2}, 1-x_{2}\right)\right\}$.

2. $\Delta^{E S S}=\left\{(0,1),\left(x_{1}, 1-x_{1}\right)\right\}$ and $\Delta^{N E} \backslash \Delta^{E S S}=\left\{\left(x_{2}, 1-x_{2}\right)\right\}$.

\section{Category III:}

1. $\Delta^{E S S}=\{(1,0)\}$ and $\Delta^{N E} \backslash \Delta^{E S S}=\{(x, 1-x)\}$.

2. $\Delta^{E S S}=\{(0,1)\}$ and $\Delta^{N E} \backslash \Delta^{E S S}=\{(x, 1-x)\}$.

Category IV: $\Delta^{E S S}=\{(x, 1-x)\}$ and $\Delta^{N E} \backslash \Delta^{E S S}=\emptyset$.

Category V: If $a=0, b \geq 0$ and $c>0$, then $\Delta^{E S S}=\{(0,1)\}$ and $\Delta^{N E} \backslash \Delta^{E S S}=\{(1,0)\}$. If $a>0, b \geq 0$, and $c=0$, then $\Delta^{E S S}=\{(1,0)\}$ and $\Delta^{N E} \backslash \Delta^{E S S}=\{(0,1)\}$. If $a=0, b<0$, and $c=0$, then $\Delta^{E S S}=\{(0,1)\}$ and $\Delta^{N E} \backslash \Delta^{E S S}=\{(1,0)\}$. If $a=0, b>0$, and $c=0$, then $\Delta^{E S S}=\{(1,0)\}$ and $\Delta^{N E} \backslash \Delta^{E S S}=\{(0,1)\}$.

\section{Category VI:}

1. $\Delta^{E S S}=\{(1,0)\}$ and $\Delta^{N E} \backslash \Delta^{E S S}=\emptyset$.

2. $\Delta^{E S S}=\{(0,1)\}$ and $\Delta^{N E} \backslash \Delta^{E S S}=\emptyset$.

Category VII: $\Delta^{N E} \backslash \Delta^{E S S}=\Delta$. 


\section{Asymptotic Stability in Replicator Dynamics}

The continuous replicator dynamics can be used to model the evolution of an infinite population. The state of the population is characterized by the vector $x(t)=\left(x_{1}, \ldots, x_{k}\right)$, where for every $m$, $x_{m}$ is the fraction of individuals playing the strategy $m$. Players are repeatedly matched with other $n-1$ players to play an $n$-player stage game. Formally, $x(t) \in \Delta$ and we may naturally interpret $x$ as a mixed strategy of a single player. A system of $k$ differential equations

$$
\dot{x}_{m}=x_{m}\left(u_{m}(x)-\sum_{l=1}^{k} x_{l} u_{l}(x)\right), m=1, \ldots, k
$$

was proposed by Taylor and Jonker [16] as a dynamical model for two-player games. For $n$-player games, replicator dynamics can be written in the following way:

$$
\dot{x}_{m}=x_{m}\left[u\left(e^{m}, x^{n-1}\right)-u\left(x, x^{n-1}\right)\right] .
$$

Properties of replicator dynamics in two-player games have been studied thoroughly. Some of them are also true for multi-player games. We will discuss these common features below. We will also present some novel behavior in three and four-player games.

\subsection{Basic theorems}

We present here without proofs some fundamental theorems which were originally formulated

for general $n$-player games and those whose proofs can be easily generalized from two-player to multi-player games. We will also show that in symmetric three-player games with two strategies, evolutionarily strategies are precisely those which are asymptotically stable in replicator dynamics.

Let us denote the set of stationary points of the replicator dynamics (18) by

$$
\Delta^{*}=\left\{x \in \Delta: u\left(e^{m}, x^{n-1}\right)=u\left(x, x^{n-1}\right) \forall_{m \in \operatorname{supp}(x)}\right\}
$$


interior stationary points by $\Delta^{* *}=\Delta^{*} \cap$ int $\Delta$, stationary points stable in the Lyapunov sense by $\Delta^{L S E}$, and asymptotically stable points by $\Delta^{A S E}$. Let $x\left(t ; x_{0}\right)$ be the solution of $(18)$ with the initial condition $x_{0}$ at time $t=0$, and $V$ and $U$ be open sets, then we have

$$
\begin{aligned}
& \Delta^{L S E}=\left\{x \in \Delta^{*}: \forall_{V \ni x} \exists_{U \ni x} \forall_{x_{0} \in U} x\left(0 ; x_{0}\right) \in U \Rightarrow \forall_{t \geq 0} x\left(t ; x_{0}\right) \in V\right\} \\
& \Delta^{A S E}=\left\{x \in \Delta^{L S E}: \exists_{V \ni x} \forall_{U \ni x} \forall_{x_{0} \in V} \exists_{T \geq 0} \forall_{t \geq T} x\left(t ; x_{0}\right) \in U\right\}
\end{aligned}
$$

Theorem $6 \Delta^{N E} \cup\left\{e^{1}, e^{2}, \ldots, e^{k}\right\} \subset \Delta^{*}$. Moreover if $x \in \Delta^{*}$ is Lyapunov stable, then $x \in \Delta^{N E}$ [17].

Theorem 7 If $x(0) \in$ int $\Delta$ and $\lim _{t \rightarrow \infty} x(t)=\hat{x}$, then $\hat{x} \in \Delta^{N E}[17]$.

The following theorem was first proved for $n=2$ in [14] and for general $n$ in [18].

Theorem 8 If $x \in \Delta^{E S S}$, then $x$ is asymptotically stable in the replicator dynamics, i.e., $x \in \Delta^{A S E}$.

Theorem 9 In supersymmetric n-player games, the average payoff is increasing along any solution of the replicator dynamics equations. It means that $\dot{u}\left(x, x^{n-1}\right) \geq 0$, and the equality holds only for $x \in \Delta^{*}$ (Prop. 3.14 in [17] and Th. 7.8.1 in [19]).

\subsection{New results}

Hofbauer and Sigmund provided an example of a symmetric two-player game with three strategies and an asymptotically stable stationary point which is not evolutionarily stable [19]. We will show that in $n$-player supersymmetric games, there is a one-to-one correspondence between asymptotically stable points and evolutionarily stable strategies.

Theorem 10 In supersymmetric n-player games, $x \in \Delta^{E S S}$ if and only if $x \in \Delta^{A S E}$.

We will adapt the proof for two-player supersymmetric games given in [19] (Th. 7.8.1), see also p.941 in [7]. 
Proof: Let $x$ be an asymptotically stable stationary point of the replicator dynamics. By Theorem $9, u\left(x, x^{n-1}\right)=u\left(x^{n}\right)$ attains a local maximum at $x$. We will show that $x$ is locally superior and therefore is evolutionarily stable. Let $x+\epsilon^{*}=\left(x_{1}+\epsilon_{1}, \ldots, x_{k}+\epsilon_{k}\right), \sum_{i=1}^{k} \epsilon_{i}=0$, be a mixed strategy in the neighborhood of $x$. If for every $i,\left|\epsilon_{i}\right|<\epsilon$ and $\epsilon$ is small enough, then

$$
u\left(\left(x+\epsilon^{*}\right)^{n}\right)<u\left(x^{n}\right) .
$$

We expand the left-hand side of (19) and obtain

$$
u\left(\left(x+\epsilon^{*}\right)^{n}\right)=u\left(x+\epsilon^{*},\left(x+\epsilon^{*}\right)^{n-1}\right)=u\left(x^{n}\right)+\sum_{k=1}^{n-1} m_{k} u\left(x^{n-k}\left(\epsilon^{*}\right)^{k}\right)+u\left(\left(\epsilon^{*}\right)^{n}\right),
$$

where $m_{k}=\left(\begin{array}{c}n-1 \\ k\end{array}\right)+\left(\begin{array}{c}n-1 \\ k-1\end{array}\right)=\left(\begin{array}{l}n \\ k\end{array}\right)$ is a binomial coefficient. It follows, for sufficiently small $\epsilon$, that there exists $0<l \leq n$ such that $u\left(x^{n-k}\left(\epsilon^{*}\right)^{k}\right)=0$ for $1 \leq k<l$ and $u\left(x^{n-l}\left(\epsilon^{*}\right)^{l}\right)<$ 0 . We also have

$$
u\left(x,\left(x+\epsilon^{*}\right)^{n-1}\right)=u\left(x^{n}\right)+\sum_{k=1}^{n-1} m_{k}^{\prime} u\left(x^{n-k}\left(\epsilon^{*}\right)^{k}\right),
$$

where $m_{k}^{\prime}=\left(\begin{array}{c}n-1 \\ k\end{array}\right)$. From (20-21), the remark after (20), and the fact that $m_{k}>m_{k}^{\prime}$ it follows, for sufficiently small $\epsilon$, that

$$
u\left(\left(x+\epsilon^{*}\right)^{n}\right)<u\left(x,\left(x+\epsilon^{*}\right)^{n-1}\right)
$$

so $x$ is locally superior and therefore evolutionarily stable. The other direction is provided by Theorem 8 .

Since any symmetric $n$-player game with two strategies is equivalent to a supersymmetric game $[9,10,11]$, we have the following corollary.

Corollary 2 In symmetric n-player games with two strategies, $x \in \Delta^{E S S}$ if and only if $x \in$ $\triangle^{A S E}$.

In particular, in Category I and II, there are two asymptotically stable Nash equilibria which are also evolutionarily stable; in all other (except the last one) categories there is a 
unique evolutionarily stable strategy which is globally asymptotically stable. Let us mention that in Category III there is a mixed Nash equilibrium which is stable from the right side and unstable from the left side and in Category $\mathrm{V}$ there is a pure unstable Nash equilibrium.

For two-player games we have

Theorem 11 If $x$ and $y$ are two different interior stationary points of two-player replicator dynamics, i.e., $x, y \in \Delta^{* *}$, then for all $\alpha, \beta \in R$ such that $\alpha x+\beta y \in \Delta$ we have $\alpha x+\beta y \in \Delta^{* *}$ [20].

This is not generally true in multi-player games; three-player games in Category II of our classification have two isolated interior Nash equilibria.

In two-player games, every interior evolutionarily stable strategy is globally asymptotically stable.

Theorem 12 In two-player games, if $\hat{x} \in$ int $\Delta \cap \Delta^{E S S}$ and if $x\left(t, x_{0}\right)$ is a solution of a replicator dynamics passing by $x_{0} \in$ int $\Delta$, then $\lim _{t \rightarrow \infty} x\left(t, x_{0}\right)=\hat{x}$ [19].

Example 1 shows that in supersymmetric 4-player games with two strategies there can be two interior evolutionarily stable strategies and hence neither of them is globally asymptotically stable. Let us also recall that games in Category II have two evolutionarily stable strategies, one pure and one interior, so again the interior one is not globally asymptotically stable.

\section{Risk-Dominance and Asymptotic Stability}

In the first two categories of our classification, there are games with two evolutionarily stable Nash equilibria. The problem of equilibrium selection is therefore not resolved. Here we will discuss the concept of risk-dominance [13] and its relation to the size of the basin of attraction of a Nash equilibrium in the replicator dynamics. 
The notion of risk-dominance was introduced and thoroughly studied by Harsányi and Selten [13]. The general theory is based on the so called tracing procedure. In symmetric two-player games with two strategies and two symmetric pure Nash equilibria, one can show that a strategy risk-dominates the other one if it has a higher expected payoff against a player playing both strategies with the probability $1 / 2$. It follows that this is equivalent to the first strategy having a bigger basin of attraction.

The procedure of Harsányi and Selten was applied by Kim [6] to n-player games with two strategies and two pure symmetric Nash equilibria (three-player games in Category I of our classification; we assume that $a>0$ and $c>0$ ). He showed that the first strategy risk dominates the second one if and only if

$$
a^{3}>2 a b c+c^{3}
$$

Now, the first strategy has a bigger basin of attraction than the second one if $x<1 / 2(x$ is the unique interior Nash equilibrium). This holds if the expected payoff of the first strategy is bigger than that of the second one against two players playing both strategies with the probability $1 / 2$. We obtain that the first strategy has a bigger basin of attraction than the second one if and only if

$$
a>2 b+c
$$

We have the following simple proposition.

Proposition 1 Let $b>0$. If the first strategy has a bigger basin of attraction than the second one, then it risk-dominates the second one.

Proof: Assume that

$$
a-c>2 b
$$

Then

$$
(a-c)\left(a^{2}+a c+c^{2}\right)>2 b\left(a^{2}+a c+c^{2}\right)
$$


hence

$$
a^{3}-c^{3}>2 a b c .
$$

If $b<0$, then of course an analogous proposition holds for the second strategy.

It is easy to see that if $b=0$ or $b=(c-a) / 2$ (in the second case the incentive function $w(x)$ in (6) is linear), then conditions (22) and (23) are equivalent and risk-dominant strategies are those with bigger basins of attraction.

However, as the following example shows, the converse proposition does not hold.

Example 2 If $a=4, b=1$, and $c=3$, then the first strategy is risk-dominant but it has a smaller basin of attraction than the second one.

The relationship between different approaches to equilibrium selection was recently discussed by Kim [6]. He provided an interpretation of the fact that in two-player games with two strategies, the risk-dominant strategy is selected by all criteria. He showed that that the equivalence of those criteria breaks down for games with more than two players.

\section{Summary}

We provided a classification of symmetric three-player games with two strategies and studied evolutionary and asymptotic stability (in the replicator dynamics) of their Nash equilibria. There exist two classes of games with two evolutionarily stable strategies. In the first one, there are two pure evolutionarily stable strategies; in the second one, there is one pure and one mixed evolutionarily stable strategy. In subsequent papers we will investigate the stochastic stability of these Nash equilibria in stochastic replicator dynamics, Kandori-Mailath-Rob and Young models and in spatial games with local interactions. The problem of the selection of a mixed Nash equilibrium is especially interesting.

Acknowledgments JM would like to thank the Polish Committee for Scientific Research for a financial support under the grant KBN 5 P03A 02520. 


\section{References}

[1] Kandori M, Mailath GJ, and Rob R (1993) Learning, mutation, and long-run equilibria in games. Econometrica 61: 29-56

[2] Blume LE (1993) The statistical mechanics of strategic interaction. Games Econ. Behav. 5: $387-424$

[3] Ellison G (1993) Learning, local interaction, and coordination. Econometrica 61: 1047-1071

[4] Young, PH (1998) Individual Strategy and Social Structure: An Evolutionary Theory of Institutions. (Princeton, Princeton University Press)

[5] Ellison G (2000) Basins of attraction, long-run stochastic stability, and the speed of stepby-step evolution. Review of Economic Studies 67: 17-41

[6] Kim Y (1996) Equilibrium selection in n-person coordination games. Games Econ. Behav. 15: $203-227$

[7] Broom M, Cannings C, and Vickers GT (1997) Multi-player matrix games. Bull. Math. Biology 59: 931-952

[8] Kobayashi J (2000) Evolutionarily stable strategy in more than two player games. Sociological Theory and Method 15: 209-216

[9] Hofbauer J, Plank M (1996) Evolutionary dynamics for binary n-person games. Preprint.

[10] Hofbauer J, Sorger G (2002) A differential game approach to evolutionary equilibrium selection. Int. Game Theory Review 4: 17-31

[11] Sandholm B (2001) Potential games with continuous player sets. J. Econ. Theory 97: 81108 
[12] Monderer D, Shapley LS (1996) Potential games. Games Econ. Behav. 14: 124-143

[13] Harsányi J, Selten R (1988) A General Theory of Equilibrium Selection in Games. (MIT Press, Cambridge, MA)

[14] Hofbauer J, Schuster P, and Sigmund K (1979). A note on evolutionarily stable strategies and game dynamics. J. Theor. Biology 81: 609-612

[15] Bishop T, Cannings C (1976) Models of animal conflict. Adv. Appl Prob 8: 616-621

[16] Taylor P, Jonker L (1978) Evolutionarily stable strategies and game dynamics. Mathematical Biosciences 40: 145-156

[17] Weibull JW (1995) Evolutionary Game Theory (MIT Press, Cambridge, MA)

[18] Palm G (1984) Evolutionarily stable strategies and game dynamics for n-person games. J. Math. Biology 19: 329-334

[19] Hofbauer J, Sigmund K (1998) Evolutionary Games and Population Dynmics (Cambridge University Press, Cambridge)

[20] Zeeman E (1980) Population Dynamics from Game Theory, in Global Theory of Dynamical Systems (Springer Verlag, Berlin) 